\title{
Téoros
}

Revue de recherche en tourisme

\section{Le rapport à la temporalité du backpacker}

\section{Jocelyn Lachance}

Volume 32, numéro 1, 2013

Tourisme des routards

URI : https://id.erudit.org/iderudit/1036656ar

DOI : https://doi.org/10.7202/1036656ar

Aller au sommaire du numéro

Éditeur(s)

Université du Québec à Montréal

ISSN

0712-8657 (imprimé)

1923-2705 (numérique)

Découvrir la revue

\section{Citer cet article}

Lachance, J. (2013). Le rapport à la temporalité du backpacker. Téoros, 32(1), 73-79. https://doi.org/10.7202/1036656ar

\section{Résumé de l'article}

La littérature scientifique insiste depuis plusieurs années sur l'hétérogénéité du groupe des backpackers, ce qui pose la question de leurs caractéristiques communes. L'article qui suit vérifie l'hypothèse selon laquelle le backpacking se caractérise par une expérience singulière de la temporalité vécue sous le signe de la maîtrise par le voyageur. Au-delà des origines sociales, culturelles et nationales diversifiées des backpackers, de leurs motivations personnelles, de leurs parcours, de leurs budgets et des pratiques qui les distinguent les uns des autres, les backpackers partageraient l'expérience d'une rupture provisoire avec les contraintes temporelles, de la création de rythmes personnels et de la réorganisation de la temporalité du voyage par le récit. En proposant une lecture exhaustive des articles scientifiques issus de la littérature anglo-saxonne, l'article révèle la place occupée par ce rapport singulier à la temporalité et montre comment elle permet de penser le groupe des backpackers au-delà de son hétérogénéité.
Ce document est protégé par la loi sur le droit d'auteur. L'utilisation des services d'Érudit (y compris la reproduction) est assujettie à sa politique d'utilisation que vous pouvez consulter en ligne.

https://apropos.erudit.org/fr/usagers/politique-dutilisation/ 


\title{
Le rapport à la temporalité du backpacker
}

\author{
Jocelyn LACHANCE \\ Chercheur postdoctoral en sociologie \\ Université de Pau, Laboratoire SET (Société, Environnement et Territoire) \\ jocelyn.lachance@univ-pau.fr
}

RÉSUMÉ: La littérature scientifique insiste depuis plusieurs années sur l'hétérogénéité du groupe des backpackers, ce qui pose la question de leurs caractéristiques communes. L'article qui suit vérifie l'hypothèse selon laquelle le backpacking se caractérise par une expérience singulière de la temporalité vécue sous le signe de la maîtrise par le voyageur. Au-delà des origines sociales, culturelles et nationales diversifiées des backpackers, de leurs motivations personnelles, de leurs parcours, de leurs budgets et des pratiques qui les distinguent les uns des autres, les backpackers partageraient l'expérience d'une rupture provisoire avec les contraintes temporelles, de la création de rythmes personnels et de la réorganisation de la temporalité du voyage par le récit. En proposant une lecture exhaustive des articles scientifiques issus de la littérature anglo-saxonne, l'article révèle la place occupée par ce rapport singulier à la temporalité et montre comment elle permet de penser le groupe des backpackers au-delà de son hétérogénéité.

Mots-clés: Backpacking, temporalité, rythme, récit, hypermodernité.

Depuis plusieurs années, le backpacking fait l'objet de recherches dans la littérature scientifique de langue anglaise, notamment dans le champ des Tourism Studies. Â travers une lecture originale, cet article propose de montrer qu'au-delà de la diversité de leurs parcours et de leurs profils, les backpackers partagent un rapport singulier à la temporalité vécu sous le signe de la maîtrise. En ce sens, notre article répond à la question suivante : derrière la multiplicité des pratiques, des motivations, des origines sociales, culturelles et nationales des différents backpackers, quelles caractéristiques fondent l'originalité de leur expérience du voyage dans le paysage du tourisme contemporain?

Par temporalité, nous entendons ici à la fois les rythmes du voyage, c'est-à-dire la vitesse des déplacements et la durée des séjours dans les différents lieux, ainsi que les rythmes constitutifs de l'expérience du backpacking, comme ceux des rencontres, des repas, du sommeil, etc. Nous y incluons aussi la dimension linéaire de la temporalité, et plus singulièrement la durée du voyage ainsi que sa place dans l'histoire biographique du voyageur. Cette définition de la temporalité, qui prend en compte plusieurs dimensions, s'accorde avec celle qu'en donnent les théoriciens de l'hypermodernité (Aubert, 2003;
Lachance, 2011). La temporalité est entendue ici comme une expérience subjective, car c'est à la fois comment ces rythmes, cette durée et ce récit sont maîtrisés et vécus par le voyageur qui est analysé. De plus, dans cette perspective hypermoderne, le sujet est en mesure de transformer ce rapport subjectif à travers des actions initiées personnellement. Il s'agit donc d'un rapport à la temporalité vécu sous le signe de la maîtrise. Comme nous le verrons, le backpacking, en tant que pratique constitutive d'un nouveau cadre d'expérience, se caractérise par ce type de rapport à la temporalité.

\section{Méthodologie}

Pour rendre compte de ce rapport original, nous proposons une lecture exhaustive des études issues de la littérature scientifique qui abordent explicitement ou implicitement cette question chez les backpackers. Nous avons concentré notre travail sur les articles de langue anglaise. Les écrits en français apparaissant plus tard et n'utilisant pas le terme backpacking en y préférant, entre autres, celui de routard, il nous semble périlleux de les amalgamer avec le corpus anglo-saxon qui a construit l'objet d'étude du backpacking. Même si certains dictionnaires reconnaissent les termes de backpacking et de 
routards comme de parfaits équivalents, un travail s'impose concernant le rapport entre ces deux notions. En utilisant l'entretien semi-directif et les histoires de vie, nombre de ces recherches s'intéressent à la représentation de voyageurs occidentaux (Cohen, 1972, 1973; Riley, 1988; Elsrud, 1998, 2001; Hampton, 1998; Desforges, 2000; Murphy, 2001; Scheyvens, 2002; Sørensen, 2003; Spreitzhofer, 1998; Noy, 2004) tandis que des travaux plus récents insistent sur l'apparition de backpackers d'origine asiatique (Teo et Leong, 2006). Ces études explorent les représentations de voyageurs de différents pays, mais leurs échantillons respectifs les distinguent les unes des autres en trois groupes. Les premières analysent une population de backpackers selon l'appartenance nationale. Ainsi, des recherches traitent spécifiquement des backpackers issus de pays anglo-saxons (Desforges, 1998, 2000; Bell, 2002; O’Reilly, 2006), de pays scandinaves (Elsrud, 1998, 2001; Enoch et Grossman, 2010) ou plus singulièrement d'origine israélienne (Uriely et al., 2002; Maoz, 2004, 2005, 2006; Noy, 2004, 2005; Noy et Cohen, 2005; Enoch et Grossman, 2010). Les secondes analysent plutôt le backpacking en interrogeant des voyageurs circulant sur une zone géographique prédéterminée en y entremêlant les origines nationales. Ces zones concernent principalement l'Océanie (Loker-Murphy et Pearce, 1995; Loker-Murphy, 1996; Vance, 2004; Cooper et al., 2004; Peel et Steen, 2007; Pearce et Foster, 2007) et l'Asie (Tsaur et al., 1997; Spreitzhofer, 1998; Hampton, 1998; Teas, 1988; Richards et Wilson, 2004; Teo et Leong, 2006; Muzaini, 2006). Les troisièmes privilégient une approche plus «globale» parce qu'elles additionnent les zones géographiques et les origines nationales ou encore parce qu'elles proposent une lecture plus générale du phénomène backpacking (Murphy, 2001; Scheyvens, 2002; Sørensen, 2003; Cohen, 2004; Maoz, 2007; Lachance, 2007, 2010a, 2010b). Malgré la diversité apparente des recherches, force est de constater que les études actuellement disponibles s'intéressent surtout aux backpackers occidentaux avec une forte présence des pays anglo-saxons à la fois à travers l'origine des voyageurs interrogés et les zones géographiques privilégiées.

Notre méthodologie consiste donc en une lecture transversale et une confrontation des études disponibles actuellement sur le phénomène backpacking, en mettant en avant les éléments qui révèlent leur rapport à la temporalité. Nous évoquerons dans un premier temps les caractéristiques autour desquelles il semble exister un certain consensus pour définir l'originalité de la pratique du backpacking dans le paysage du tourisme. Il est à noter que la littérature scientifique de langue anglaise sur le backpacking est traversée par la question du rapport du backpacker au monde du tourisme. Sans entrer ici dans le débat, notons que si le backpacking semble considéré comme une forme de tourisme par la plupart des auteurs, ces mêmes auteurs observent généralement que les backpackers se distinguent, dans leurs discours, de la figure du touriste. Nous verrons dans un deuxième temps que, au-delà de ces caractéristiques, il existe une autre dimension caractérisant l'expérience du backpacking : il s'agit d'un rapport singulier à la temporalité, un rapport vécu notamment sous le signe de la maîtrise.

\section{Motivations partagées par les backpackers}

En cherchant l'origine du backpacking, certains penseront peut-être aux errances de la Beat Generation, illustrée dans le célèbre On the Road de Jack Kerouac (1957), ou aux voyages initiatiques inhérents au mouvement hippie. Or, les formes de voyage connues au sein de ces deux mouvements sont rarement interprétées comme des précurseurs du backpacking, entre autres parce que le contexte social semble affecter profondément la signification que prend le voyage aux yeux des différentes générations. Comme le soulignent Frédéric Monneyron et Martine Xiberras (2008), les voyages, même individuels, au sein du mouvement hippie s'inscrivent dans une participation à un mouvement collectif et dans une volonté de changer le monde. Le backpacking, quant à lui, n'est associé à aucun mouvement collectif, à priori, et le backpacker ne chercherait, au contraire, qu'à transformer son monde, c'est-à-dire sa vision sur le monde et sa propre personne. D'autres chercheurs font parfois remonter l'origine du backpacking au Grand Tour du $18^{e}$ siècle en Europe. Si le Grand Tour s'apparente alors à un temps de passage institué par les classes dominantes, en revanche, à sa pratique correspond l'émergence d'un nouveau discours du voyageur sur son entreprise. En effet, comme le remarque Chloe Chard (1999), les journaux et les lettres des jeunes s'adonnant au Grand Tour au $18^{\mathrm{e}}$ siècle montrent que le voyage est déjà représenté comme une aventure personnelle parsemée de transgressions et de prises de risques (Chard, 1999 : 167). En d'autres termes, malgré l'apparence d'un voyage "programmé», inscrit dans le cadre d'une destinée tracée pour les plus jeunes, l'expérience du Grand Tour est souvent vécue comme le temps d'une aventure singulière. C'est ici sans doute l'argument le plus important pour considérer le Grand Tour comme un précurseur du backpacking dont les adeptes revendiquent souvent l'originalité de leur expérience. Notons que certains films récents viennent alimenter la représentation et l'imaginaire du backpacking, dont The Beach, de Danny Boyle (2000), qui s'inspire du roman éponyme d'Alex Garland, Hostel, d'Eli Roth (2005), dans un style près de celui de Quentin Tarantino, et, plus récemment, Into the Wild, de Sean Penn (2007). Les figures identificatoires des backpackers dans la littérature ont d'ailleurs fait l'objet d'une recherche (Richards et Wilson, 2004).

Dans les champs des Tourism Studies, les études sur le backpacking sont généralement traversées par la tentative de définir les caractéristiques communes de voyageurs qui forment cependant un groupe hétérogène étant donné la diversité de leurs profils et de leurs pratiques. Toutefois, depuis les recherches d'auteurs comme Vogt (1976) et Cohen (1972, 1973, 1979) - que plusieurs chercheurs considèrent comme les précurseurs des études sur le backpacking - trois caractéristiques sont récurrentes et persistent dans les écrits des spécialistes jusqu'à aujourd'hui.

\section{La recherche $d^{\prime}$ un sentiment de liberté}

Les études de Vogt (1976) et de Cohen (1972, 1973, 1979) pointaient déjà cette dimension, notamment en mettant en avant la volonté du sujet à voyager en dehors des circuits organisés et des destinations privilégiées par les touristes. Bien que le terme backpacker s'impose dans la littérature seulement 
au cours des années 1990, Vogt (1976) et Cohen (1972, 1973, 1979) sont les premiers à pointer l'émergence d'un nouveau type de voyageur caractérisé, entre autres, par sa recherche d'indépendance vis-à-vis de l'industrie touristique. Les notions de liberté et de mobilité apparaissent dans les travaux de Vogt et de Cohen comme étant centrales pour comprendre les motivations de ce voyageur indépendant qui fuit les circuits organisés. Dès cette époque, les termes employés pour désigner ces voyageurs sont nombreux et diversifiés : drifters — vagabonds (Cohen, 1972); nomads — nomades (Cohen, 1973); wanderers - aventuriers (Vogt, 1976). Il est intéressant de noter que les noms donnés par les auteurs à cette forme de voyage renvoient à la mobilité et principalement au rapport du voyageur à l'espace.

Cette dimension de la liberté semble donc, avant tout, être associée intimement à une volonté de distinction par rapport au tourisme de masse, une remarque qui traverse d'ailleurs les études sur le backpacking jusqu'à aujourd'hui. Ce sentiment subjectif de liberté s'expliquerait à travers plusieurs facteurs, dont la fuite des responsabilités inhérentes à la vie quotidienne (Desforges, 2000 : 934) et le dégagement des contraintes liées aux besoins matériels (Elsrud, 1998 : 315). Certains facteurs explicatifs renvoient explicitement à un rapport singulier à la temporalité expérimentée au cours de la pratique du backpacking: le fait de différer dans le temps l'entrée dans l'âge adulte (Noy, 2004 : 84; Lachance, 2010a : 55 ) et la création d'un temps propice aux expérimentations sous toutes ses formes (Desforges, 2000 : 935; Elsrud, 2001 : 599; Lachance, 2007 : 38).

\section{La recherche d'authenticité}

La question de l'authenticité est centrale dans le champ des Tourism Studies et est récurrente dans le discours des backpackers (Bruner, 1991; Murphy, 2001; Richards et Wilson, 2004). Avec l'apparition d'études qualitatives sur le backpacking, la place occupée par l'authenticité s'incarne dans la parole des acteurs qui affirment que sa recherche constitue une des principales motivations de leur voyage (Elsrud, 2001 : 601; Noy, 2004 : 85). Cette quête d'authenticité s'exprime dans divers aspects du voyage de forme backpacking. Un premier argument consiste à dire que la rencontre avec l'autre, les locaux (Noy, 2005 : 148), mais également avec les autres backpackers, est plus «naturelle» et plus «vraie» dans le contexte du voyage. Dans le cas de la rencontre avec les locaux, cet argument repose parfois sur le «mythe du bon sauvage» et sur un fantasme de retour à la nature, voire aux racines de l'existence. Certains backpackers vont même jusqu'à adopter la mode locale pour ressembler aux populations visitée (Muzaini, 2006). Dans le cas de la rencontre avec les autres backpackers, l'authenticité reposerait sur le caractère ponctuel et éphémère des rencontres, et sur la facilité de s'engager et de se désengager dans la relation avec l'autre (Sørensen, 2003 : 854). En d'autres termes, elle reposerait sur une temporalité relationnelle singulière qui trouverait sa place au sein du voyage de forme backpacking. Dans ce contexte, rester avec l'autre pour partager, par exemple, quelques kilomètres le temps d'un déplacement ou quelques jours le temps d'un séjour dans un village signifierait l'expression du sujet de vouloir prolonger ce temps réuni (Lachance, $2007: 36$, et 2010a : 58). La relation serait authentique, car, dans le contexte du backpacking, il n'existerait jamais de relation forcée ou imposée par des contraintes extérieures au sujet. Par ailleurs, l'authenticité touche aussi la relation que le sujet entretient avec lui-même, dans la mesure où le voyage lui servirait à révéler des aspects inédits, mais authentiques de lui-même (Noy, 2004 : 85-86).

La capacité d'adaptation et la mise à l'épreuve de soi Les études montrent aussi que le backpacker valorise sa capacité d'adaptation aux différentes situations qu'offre la route. L'adaptation aux langues locales, aux codes culturels, à l'alimentation, au climat et au mode de vie en général est mise en avant comme une qualité du backpacker. D'une part, cette adaptabilité marquerait la volonté du sujet à s'ouvrir à l'autre et au monde (Lachance, 2007 : 37). D'autre part, l'adaptation est perçue comme une réponse à des épreuves que le sujet affronte tout au long de son voyage. De nombreuses études pointent d'ailleurs la place occupée par la prise de risque prenant la forme de la maladie et de l'accident motorisée, mais aussi de la confrontation à l'inattendu et à l'altérité culturelle (Elsrud, 2001 : 604 ; Noy, 2004 : 87). D'autres affirment que la capacité à répondre à des situations d'urgence est reconnue par ces voyageurs. L'adaptabilité prendrait alors le visage de la capacité du sujet à surmonter des épreuves et ces épreuves constitueraient pour plusieurs la condition même d'un voyage réussi (Elsrud, 2001 : 603). Dans une perspective socioanthropologique, cette épreuve est analysée comme une constituante du rite de passage à l'âge adulte (Vogt, 1976; Riley, 1988; Desforges, 2000; Bell, 2002; Sørensen, 2003 ; Noy, 2004). En effet, pour plusieurs auteurs, la traversée d'une telle épreuve, qu'elle soit réelle ou symbolique, participerait de la construction d'un sentiment de transformation ontologique chez le voyageur (Elsrud, 2001 : 605; Noy, 2004 : 80; Lachance, 2010a: 61). En d'autres termes, le voyage compris comme une épreuve symbolique marquerait le temps biographique du voyageur, en séparant un avant d'un après.

Or, il existe une autre caractéristique récurrente dans l'expérience du backpacking. Il s'agit de la volonté pour ces voyageurs de maîtriser la temporalité de leur voyage, ce qui se traduit par la maîtrise des rythmes, de la durée du voyage et du récit construit à partir de son expérience. En effet, l'approfondissement du corpus actuellement disponible sur le phénomène montre que la question de la temporalité est omniprésente et qu'elle autorise une lecture originale de cette forme de voyage. Cette question de la temporalité est d'ailleurs déjà évoquée dans les études, notamment dans celle de Pamela Riley (1988), lorsqu'elle relève que les voyageurs affirment que l'abondance de temps libre est une caractéristique de leur pratique, et dans celle de Torun Elsrud (1998), qui montre que la création d'un rapport original à la temporalité est l'une des expériences les plus centrales pour des femmes backpackers. Nous verrons maintenant que ce rapport singulier à la temporalité, sous le signe de la maîtrise, se développe en trois temps : la rupture provisoire avec les contraintes temporelles, la création de rythmes personnels et la réorganisation de la temporalité du voyage par le récit. 


\section{Rupture provisoire avec les contraintes temporelles} Les études sur le backpacking montrent que ses adeptes entament généralement leurs voyages à une période de transition dans leur vie, ce qui, généralement, ne semble pas être le cas pour les vacanciers et les touristes. À leur départ correspond alors l'obtention récent d'un diplôme, la fin d'un contrat de travail, parfois même une rupture amoureuse (Riley, 1988 : 313; Desforges, 2000 : 933; Sørensen, 2003 : 848). Dans son étude sur les femmes suédoises, Torun Elsrud (1998) montre que celles d'un certain âge s'adonnent aussi au backpacking au cours d'une période de transition dans leur vie (divorce, déménagement, questionnement professionnel, etc.).Leur départ se caractérise de plus par une planification minutieuse qui motive le choix de la date de départ et qui implique aussi une évaluation de ses coûts et l'élaboration d'un budget. Chez ces voyageurs indépendants, l'habileté à préparer un voyage est d'ailleurs considérée comme une qualité centrale. En d'autres termes, c'est généralement pendant une période caractérisée par la difficulté à se projeter dans l'avenir ou qui demande une prise de décision pour poursuivre dans une voie ou une autre que des personnes optent pour la pratique du backpacking. C'est pourquoi, pour des chercheurs comme Torun Elsrud, ces voyageurs s'offrent le luxe de prendre du temps sans perdre de temps dans une société qui abhorre la perte de temps (Elsrud, 1998 : 322). Ceci est représentatif de l'expérience du sujet hypermoderne qui cherche à maîtriser son rapport à la temporalité (Aubert, 2003). Les backpackers différeraient, en quelque sorte, le moment de prendre des décisions quant à leur avenir. Ils échapperaient ainsi provisoirement à l'impératif de se projeter dans l'avenir à long terme et se replieraient sur d'autres horizons temporels délimités à l'intérieur de la durée du voyage.

Le backpacking implique aussi une rupture avec les contraintes sociales qui imposent un horaire au sujet. La pratique du backpacking suppose une distance importante avec une existence rythmée par l'horaire de l'école ou du travail (Elsrud, 1998 : 327; Lachance, 2010a : 60). Les relations amicales et parentales sont d'ailleurs suspendues : elles se poursuivent généralement par l'intermédiaire d'Internet, mais elles n'imposent plus dans le quotidien de la personne des contraintes pour se rencontrer et partager du temps. Autrement dit, ce qui rythmait l'existence dans le pays natal laisse place à une nouvelle liberté pour le sujet qui peut rythmer autrement, et selon son caprice, ses journées. À l'opposé, le tourisme de masse implique plutôt de quitter les rythmes du quotidien pour suivre de nouveaux rythmes imposés par le guide et le groupe. Cet abandon des repères temporels s'expriment chez plusieurs backpackers par l'abandon concret des instruments de mesure du temps. En étudiant le cas singulier des femmes en Suède, Torun Elsrud observe qu'elles consultent moins leurs montres durant leur voyage (Elsrud, 1998 : 331). Ce geste peut alors être vécu comme le symbole d'un choix personnel et participe d'un sentiment d'abandon des repères temporels du quotidien.

Le moment du retour, et donc la durée du voyage, est aussi assujetti au choix du backpacker. Deux cas de figure sont révélés à travers les différentes recherches. Certains backpackers fixent, avant même leur départ, le moment de leur retour (Sørensen,
2003 : 853); d'autres vont plutôt déterminer ce moment plus tard, ce qui contribue, semble-t-il, à l'élaboration de leur sentiment de liberté (Murphy, 2001 : 55). Le touriste de masse ne partage pratiquement jamais cette caractéristique avec le backpacker. Dans plusieurs cas, on remarque également qu'un lien semble exister entre l'argent disponible et la durée du voyage, c'est-à-dire que les études dépeignent un voyageur qui s'efforce d'étirer la durée de son aventure en dépensant un minimum d'argent (Teas, $1988: 36$ ), ce rapport à l'argent constituant d'ailleurs pour plusieurs auteurs une caractéristique du backpacking (Riley, 1988 : 320; Loker-Murphy et Pearce, 1995 : 820; Teas, 1988 : 36). Derrière cet usage spécifique de l'argent se dessine aussi une relation avec la quête d'authenticité (payer le prix local, vivre comme la population locale, etc.) (Riley, 1988 : 321) qui le distingue une fois de plus du touriste de masse. Dans tous les cas, la durée du voyage est contrôlée et maîtrisée de diverses manières par le backpacker.

Ces premières remarques montrent que la rupture avec les contraintes temporelles s'effectue sous le signe du choix de la part de la personne qui peut éventuellement élaborer un sentiment de maîtrise autour de cette rupture provisoire. Contrairement aux périodes de transition, marquée par l'insécurité et par l'incertitude, la décision d'ouvrir la parenthèse du voyage vient possiblement changer la position du sujet face à son existence, évacuant un éventuel sentiment d'hétéronomie. Cette expérience d'un choix et d'une action ayant une incidence sur le rapport à la temporalité du sujet est représentative de ce que l'on observe dans le contexte hypermoderne (Aubert, 2003). Notons que cette parenthèse, le temps du voyage, est pour plusieurs auteurs associée au temps liminaire du rite de passage à l'âge adulte bien connu dans les sociétés traditionnelles. Pour Torun Elsrud, il s'agit d'un time bubble, un temps dont la durée est maîtrisée subjectivement par le voyageur (Elsrud, 2001 : 605).

\section{Création de rythmes personnels}

La rupture avec les contraintes temporelles de la vie ordinaire n'entraînerait pas, semble-t-il, un sentiment de horstemps chez le backpacker (Elsrud, 1998 : 317). Au contraire, il s'agit alors pour lui de remplacer ces contraintes temporelles par de nouveaux rythmes de vie dont il sera l'instigateur. Contrairement au touriste de masse qui s'en remet à une agence, à un itinéraire tracé, à des réservations, etc., le backpacker vit son expérience du voyage avec le sentiment de choisir les rythmes de son voyage et de les modifier au besoin. À ce sujet, des études sur le backpacking soulignent que la vitesse des déplacements et la durée des séjours dans les différents lieux semblent varier d'un voyageur à un autre. En revanche, des auteurs remarquent, à l'opposé, que les lieux fréquentés et les parcours empruntés par les backpackers diffèrent peu les uns des autres (Cooper et al., 2004). En d'autres termes, non seulement les rythmes du voyage sont décidés par le voyageur, mais ils seraient aussi un moyen de singulariser son parcours. D'ailleurs, la possibilité d'étirer le temps d'un séjour apparaît comme un moyen de distinction par rapport au tourisme de masse (Peel et Steen, 2007 : 1064), et le rythme des déplacements est l'un des sujets de prédilection situés au cœur des discussions entre backpackers (Sørensen, 2003 : 851). La 
décision de rester dans une ville un peu plus longtemps que prévu ou de précipiter son départ participe alors du sentiment de maîtriser les rythmes de son voyage.

Les modalités de la rencontre avec l'autre renforcent cette hypothèse d'un rythme créé par le sujet lors de la pratique du backpacking. La rencontre reste, pour ces voyageurs, essentielle et significative. Toutefois, la rencontre semble plus importante que la relation développée avec l'autre dans la mesure où la durée d'un échange dans le temps n'est pas un gage de la qualité et de l'importance d'une relation. Selon les auteurs, dans le monde des backpackers, l'engagement envers l'autre s'effectue sur la base d'un contrat implicite et constamment renouvelable minute après minute (Sørensen, 2003 : 861; Lachance, 2007 : 39). Autrement dit, une rencontre brève et éphémère, vécue cependant avec intensité par le sujet, peut devenir significative pour lui et venir s'inscrire dans le récit de son voyage. Par conséquent, ces auteurs dépeignent un contexte où le désengagement des uns et des autres est légitimé à travers le statut du backpacker qui cherche à vivre une expérience originale, unique et dépourvue de contraintes. Anders Sørensen observe d'ailleurs qu'Internet est utilisé dans cette perspective par les backpackers qui se séparent et se réunissent plus facilement, en se rencontrant par hasard et en organisant, sur une base flexible, des retrouvailles ponctuelles (Sørensen, 2003 : 860). C'est pourquoi les communautés de backpackers sont mouvantes, en perpétuelle reconstruction, au fil du hasard des arrivées, des rencontres et des séparations, et que ces communautés conservent un caractère informel (Murphy, 2001 : 65; Riley, 1988 : 324). Par contre, le temps partagé entre deux voyageurs est considéré comme intense et significatif, car, dans le contexte du backpacking, ce temps partagé serait, par définition, désiré et voulu par chaque voyageur susceptible de rompre à tout moment ce partage (Lachance, 2007 : 40). Les relations qui participent à la construction du rythme du voyage ne sont donc pas perçues comme des contraintes, mais bien comme des moyens d'affirmer sa liberté de choix et de vivre des échanges voulus, et souvent perçus comme étant authentiques.

Les rythmes créés par le backpacker ne s'effectuent pas seulement sur la base des déplacements et des relations ponctuelles. Pour plusieurs, les rythmes du corps deviennent aussi des repères, certains mangeant lorsqu'ils ont faim et dormant lorsqu'ils sont épuisés, indépendamment des horaires conventionnels et des rites qui organisent les temps des repas et du sommeil dans les différents contextes culturels. Pour d'autres encore, il s'agit de prendre les besoins corporels comme repères afin de les combattre, notamment en s'imposant un certain ascétisme alimentaire pour moins dépenser d'argent. Par exemple, certains vont dormir le jour dans des parcs pour éviter les auberges de jeunesse la nuit ou vont parfois préférer voyager pendant de longues heures, voire des jours, malgré l'épuisement qu'entraînent de telles entreprises (Elsrud, 2001 : 609). Ce rapport à la temporalité semble aussi distinguer le backpacker du touriste de masse.

Dans ces différents exemples, le sujet crée des rythmes qui sont vécus sous le signe de la maîtrise, voire de l'autonomie, notamment parce qu'ils sont subjectivement choisis et déterminés par le sujet qui vit alors une expérience radicale de désynchronisation par rapport aux rythmes connus dans le pays d'origine. Les horaires de repas, de l'école ou du travail, les rendez-vous avec les amis ou la famille, même l'alternance des jours et des nuits ne sont plus les premiers repères qui rythment l'existence du voyageur. L'improvisation est plus que jamais au rendez-vous, et le désengagement envers l'autre, mais aussi envers ses propres choix effectués la veille, trouvent ici un cadre d'interprétation nouveau qui les légitime. Notons d'ailleurs que les rythmes du voyage sont, en quelque sorte, brusqués par le backpacker qui s'autorise à changer ses plans. Ainsi, les parcours empruntés se dessineraient à partir du ressenti subjectif du voyageur, son sentiment qu'il devrait rester ou partir, s'investir dans une relation ou y mettre un terme, manger maintenant ou jeûner encore quelques heures, etc. Il semble d'ailleurs que la relation à l'attente se transforme dans le contexte du voyage de forme backpacking. Par exemple, si les retards des transports publics à la maison sont généralement mal vécus (impatience, stress, etc.), dans le contexte du backpacking, ces mêmes types de retard semblent lus autrement et vécus plutôt comme une expérience culturelle.

\section{Réorganisation de la temporalité du voyage par le récit}

Le besoin de se raconter est une autre caractéristique omniprésente dans les études sur le backpacking. Des études insistent sur la tenue de journaux de voyage par les backpackers (Richards et Wilson, 2004) et d'autres explorent, sans surprise, l'usage abondant d'Internet par la communauté des backpackers (Sørensen, 2003 : 861; Enoch et Grossman, 2010 : 521).

On sait depuis Paul Ricoeur que le récit est, pour le sujet, un moyen de réorganiser la temporalité des évènements vécus (Ricoeur, 1985). En effet, le récit d'un événement suppose toujours la sélection consciente et inconsciente de fragments de ce qui a été vécu pour en faire une histoire intelligible et partageable avec les autres. Cette opération de sélection suppose donc de donner une certaine importance à des moments singuliers d'un événement et d'en rayer d'autres de son récit. En d'autres termes, le récit d'un événement est une reconstruction narrative à partir du vécu. Ainsi, certains moments peuvent prendre une place plus ou moins importante en comparaison avec ce qui a été réellement vécu. En racontant son voyage, le backpacker s'adonne à une réorganisation de la temporalité de son aventure.

Dans les études sur le backpacking, nous pouvons repérer deux types de récit. Dans un premier temps, les recherches font état des récits qui accompagnent le déroulement du voyage. Ces récits s'organisent lors de la rencontre avec d'autres voyageurs, dont le sujet principal est souvent le voyage lui-même, mais aussi par l'intermédiaire d'Internet (Sørensen, 2003 : 860; Enoch et Grossman, 2010 : 525) et des forums (Adkins et Grant, 2007 : 194). Ces récits se caractérisent par leur évolution dans le temps du voyage. Ils témoignent à la fois des expériences vécues quotidiennement et de la transformation de la vision du monde du backpacker. Plusieurs raisons motiveraient cette prise de parole : la volonté de rencontrer d'autres voyageurs (Riley, 1988 : 324), le besoin de faire reconnaître la valeur de son voyage (O'Reilly, 2006 : 1001), voire de gagner un certain prestige au sein de la communauté des 
backpackers (Sørensen, 2003 : 856). Cette volonté de partager le récit de son voyage expliquerait même, dans certains cas, le regroupement des backpackers avec d'autres voyageurs de même nationalité avec qui il serait plus facile de communiquer son expérience (Cohen, 2004 : 47; Noy, $2004: 80$ ). Des études insistent d'ailleurs sur l'importance du statut du backpacker au sein de cette communauté de voyageurs (Sørensen, 2003 : 856).

Dans un deuxième temps, des études explorent les récits des backpackers lors de leur retour au point d'origine. Certaines d'entre elles insistent sur l'opération de formalisation de leur discours en fonction de leurs interlocuteurs. C'est, par exemple, ce que montre Luke Desforges en insistant sur la difficulté des backpackers à raconter leur histoire à de futurs employeurs (2000 : 940). Toutefois, la plupart des recherches pointent surtout l'importance du récit pour raconter et partager une expérience significative. Cette expérience s'accompagne d'un discours qui redéfinirait le voyage comme un temps de transformation ontologique du sujet (Noy, 2004 : 78), qui séparerait l'avant et l'après-voyage. Autrement dit, le récit permettrait aussi d'insérer l'expérience du voyage dans l'histoire biographique du sujet. Ce «mythe de passage», pour reprendre l'expression de l'anthropologue Frank Michel, orienterait d'ailleurs la construction du récit de voyage et donc de sa temporalité $(2004,2006)$. Par exemple, les épisodes de prise de risques, semblant représenter objectivement une place minime dans le temps du voyage, prennent souvent une place centrale dans le récit des voyageurs et participent à magnifier leurs parcours (Elsrud, 2001 : 604; Tsaur et al., 1997 : 1050). Ainsi la réorganisation de la temporalité du voyage par le récit s'effectue par la sélection d'évènements significatifs aux yeux de ces voyageurs et est influencée par la volonté de valoriser le voyage comme un temps de transformation ontologique. Encore ici, c'est une expérience singulière de la temporalité que le backpacker vit, une expérience qui s'exprime cette fois sous la forme de la parole et toujours sous le signe de la maîtrise.

\section{Conclusion}

Depuis longtemps, le backpacking s'est présenté comme un objet d'étude difficile à saisir, entre autres parce que cette forme de voyage se révèle à travers une multiplicité de pratiques et une diversité de profils de voyageurs. Notre lecture montre que, à travers la notion de backpacking, les chercheurs ont pointé une forme de voyage qui pose la question de ce qui rassemble des voyageurs se distinguant cependant sur plusieurs plans. Au-delà de l'hétérogénéité du groupe des backpackers, notre article montre que les études scientifiques qui ont construit puis développé la réflexion sur le backpacking révèlent parfois explicitement, souvent implicitement, que l'expérience de cette forme de voyage se caractérise par un rapport singulier du voyageur à la temporalité. Aux antipodes du touriste de masse qui s'en remet à une instance extérieure pour réguler son temps, le backpacker se pose dans une position de maitrise de la temporalité. Certes, sans doute existe-t-il dans le paysage du voyage contemporain des positions mitoyennes entre le touriste de masse et le backpacker, ce qui peut brouiller les frontières entre les différentes figures de voyageur. Toutefois, à travers la littérature scientifique, force est de constater que le backpacker ne rompt pas seulement avec les contraintes temporelles du quotidien, il ne se créé pas seulement des rythmes personnels et ne réorganise pas seulement la temporalité de son voyage par le récit. Il exprime et revendique, à travers le discours recueilli par les différents chercheurs, cette rupture, cette création et cette réorganisation, ce qui le rapproche de la figure du sujet hypermoderne, maitre de la temporalité.

Récemment, des recherches abordent le rôle de plus en plus important d'Internet et du téléphone portable dans l'expérience du backpacker (Paris, 2011, 2012). Paris propose ainsi de distinguer la figure du backpacker de celui du flashpacker, ce dernier se caractérisant par un usage des technologies de l'information et de la communication (téléphone portable, téléphone intelligent, ordinateur portable, tablette, etc.) pendant son voyage. Il serait intéressant dans l'avenir de vérifier comment l'usage de ces technologies joue un rôle dans le rapport à la temporalité du voyageur. En effet, des études montrent déjà que l'usage de ces technologies dans la vie des personnes transforme leur rapport à la temporalité (Jauréguiberry, 2003; Lachance, 2011). Reste à voir si ces usages, dans le contexte du voyage, renforcent ce que nous avons observé dans la littérature scientifique analysant le backpacking : un sujet cherchant à maîtriser la temporalité, et qui utiliserait les technologies récentes dans cette perspective. Nous assisterions alors à l'émergence de la figure du voyageur hypermoderne, un individu nostalgique de l'expérience liminaire propre au voyage moderne dans un monde désormais encombré par les technologies de l'information et des communications.

\section{Références}

ADKINS, Barbara et Eryn GRANT (2007) «Backpackers as a community of strangers: The interaction order of an online backpacker notice board», Qualitative Sociology Review, vol. 3, p. 188-201.

AUBERT, Nicole (2003) Le culte de l'urgence. La société malade du temps, Paris : Flammarion. 375 p.

BELL, Claudia (2002) «Young New Zealand travellers as secular pilgrim.», Tourist Studies, Londres: Sage, vol. 2, p. 143-158.

BOYLE, Danny (2000) The Beach, film, États-Unis : Figment Films, DVD, 119 min.

BRUNER, Edward M. (1991) «Transformation of self in tourism», Annals of Tourism Research, vol. 18, p. 238-250.

CHARD, Chloe (1999) Pleasure and Guilt on the Grand Tour: Travel Writing and Imaginative Geography, 1600-1830, Manchester : Manchester University Press. 256 p.

COHEN, Erik (1972) «Toward a Sociology of International Tourism», Social Research, vol. 39, p. 164-182.

COHEN, Erik (1973) «Nomads from Affluence: Notes on the Phenomenon of Drifter Tourism. International», Journal of Comparative Sociology, vol. 14, p. 89-103.

COHEN, Erik (1979) «A Phenomenology of Tourist Types», Sociology, vol. 13, p. 179-201.

COHEN, Erik (2004) «Backpacking: Diversity and change», DANS RICHARDS, Greg et Julie WILSON (sous la direction de), The global nomad, p. 43-59. Clevedon : Channel View.

COOPER, Malcom; Kieran O'MAHONEY et Patricia ERFURT (2004) «Backpackers join the mainstream? An analysis of backpacker 
employment on the "Harvest trail circuit" Australia », DANS », DANS RICHARDS, Greg et Julie WILSON (sous la direction de), The global nomad, p. 180-195. Clevedon : Channel View.

DESFORGES, Luke (1998) «"Checking out the planet": Global Representations/Local Identities and Youth Travel», DANS SKELTON, Tracey et Gill VALENTINE (sous la direction de), Cool Places: Geographies of Youth Culture, p. 175-192. London : Routledge.

DESFORGES, Luke (2000) «Traveling the world: Identity and travel biography», Annals of Tourism Research, vol. 27, p. 926-945.

ELSRUD, Torun (1998) «Time Creation in Traveling: The Taking and Making of Time among Women Backpackers», Time and Society, vol. 7, p. 309-334.

ELSRUD, Torun (2001) «Risk Creation in Traveling: Backpacker Adventure Narration", Annals of Tourism Research, vol. 28, p. 597-617.

ENOCH, Yael et Ronit GROSSMAN (2010) «Blogs of Israeli et Danish Backpackers to India ", Annals of Tourism Research, vol. 37, $\mathrm{n}^{\circ} 2$, p. 520-536.

HAMPTON, Mark (1998) «Backpacker Tourism and Economic Development», Annals of Tourism Research, vol. 25, p. 639-660.

JAUREGUIBERRY, Francis (2003) Les branchés du portable. Sociologie des usages, Paris : PUF, Coll. «Sociologie d'aujourd'hui». 196 p.

KEROUAC, Jack (1957) On the Road, New York : Viking Press. 320 p.

LACHANCE, Jocelyn (2007) «Le backpacking : un visage positif de l'errance», DANS DUPONT, Sébastien et Jocelyn LACHANCE (sous la direction de), Errance et solitude chez les jeunes, p. 35-42. Paris : Tétraèdre.

LACHANCE, Jocelyn (2010a) «Le Backpacking : voyager hors de soi», DANS DUCOURNAU, Nicolas; Louis MATHIOT; Meryem SELLAM et Jocelyn LACHANCE (sous la direction de), La recherche d'extase chez les jeunes, p. 55-62. Québec: Presses de l’Université Laval.

LACHANCE, Jocelyn (2010b) «Traveler», DANS LE BRETON, David et Daniel MARCELLI (sous la direction), Dictionnaire de l'adolescence et de la jeunesse, p. 862-863. Paris : Presses Universitaires de France.

LACHANCE, Jocelyn (2011) L'adolescence hypermoderne. Le nouveau rapport au temps des jeunes, Québec: Presses de l'Université Laval. 149 p.

LOKER-MURPHY, Laurie (1996) «Backpackers in Australia: A Motivationbased Segmentation Study», Journal of Travel and Tourism Marketing, vol. $5, n^{\circ} 4$, p. 23-45.

LOKER-MURPHY, Laurie et PEARCE, Philip L. (1995) «Young Budget Travelers: Backpackers in Australia ", Annals of Tourism Research, vol. 22, p. 819-843.

MAOZ, Darya (2004) «The Conquers and the Settlers: Two Groups of Young Israeli Backpackers», DANS RICHARDS, Greg et Julie WILSON (sous la direction de), The global nomad, p. 109-122. Clevedon : Channel View.

MAOZ, Darya (2005) «Young Adult Israeli Backpackers in India», DANS NOY, Chaim et Erik COHEN (sous la direction de), Israeli Backpackers: From Tourism to Rite of Passage, p. 159-186. Albany : State University of New York Press.

MAOZ, Darya (2006) «The mutual gaze», Annals of Tourism Research, vol. 33, $\mathrm{n}^{\circ} 1, \mathrm{p} .221-239$.

MAOZ, Darya (2007) «Backpackers' Motivations: The Role of Culture and Nationality", Annals of Tourism Research, vol. 34, n 1, p. 122-140.

MICHEL, Franck (2004). Désirs d'Ailleurs. Essai d'anthropologie des voyages, Québec : Presses de l'Université Laval. 367 p.

MICHEL, Franck (2006) «Rites de voyages et mythe de passage», L'Autre Voie, $\mathrm{n}^{\circ} 2<\mathrm{http} / / /$ www.deroutes.com/Rites.htm>, consulté le 3 septembre 2013.

MURPHY, Laurie (2001) «Exploring Social Interactions of Backpackers», Annals of Tourism Research, vol. 28, p. 50-67.

MUZAINI, Hamza (2006) «Backpacking Southeast Asia: Strategies of looking local», Annals of Tourism Research, vol. 33, nº 1, p. 144-161.
MONNEYRON, Frédéric et Martine XIBERRAS (2008) Le monde hippie de l'imaginaire psychédélique à la révolution informatique, Paris : Imago. $166 \mathrm{p}$.

NOY, Chaim (2004) «The Trip Really Changed Me: Backpackers' Narrative of Self-Change", Annals of Tourism Research, vol. 31, p. 78-102.

NOY, Chaim (2005) «Israeli Backpackers: Narrative, Interpersonal Communication, and Social Construction", DANS NOY, Chaim et Erik COHEN (sous la direction de), Israeli Backpackers: From Tourism to Rite of Passage, p. 111-157. Albany : State University of New York Press.

NOY, Chaim et Erik COHEN (2005) Israeli Backpackers: From Tourism to Rite of Passage. Albany : State University of New York Press. 267 p.

O'REILLY, Camille (2006) «From drifter to gap year tourist: Mainstreaming backpacker travel», Annals of Tourism Research, vol. 33, nº 4, p. 998-1017.

PARIS, Cody M. (2011) «Understanding the statusphere and blogosphere: An analysis of virtual backpacker spaces", DANS LAW, Rob et Matthias FUCHS (sous la direction de), Information and communication technologies in tourism 2011: Proceedings of the international, p. 443-455. Innsbruck : AUSTRIA.

PARIS, Cody M. (2012) «Flashpackers: An Emerging Sub-Culture?», Annals of Tourism Research, vol. 39, n² 2, avril, p. 1094-1115.

PEARCE, Philip et Faith FOSTER (2007) "A "University of Travel": Backpacker Learning”", Tourism Management, vol. 28, n 5, p. 1285-1298.

PEEL, Victoria et Adam STEEN (2007) «Victims, hooligans and cash-cows: Media representations of the international backpacker in Australia ", Tourism Management, vol. 28, $\mathrm{n}^{\circ}$ 4, p. 1057-1067.

PENN, Sean (2007) Into the Wild, film, États-Unis: Paramount Vantage, DVD, $147 \mathrm{~min}$.

RICHARDS, Greg et Julie WILSON (2004) «Travel writers and writers who travel: Nomadic Icons for the backpacker subculture?», Journal of Tourism and Cultural Change, vol. 2, $\mathrm{n}^{\circ} 1$, p. 46-68.

RICOEUR, Paul (1985) Temps et récits, Paris : Le Seuil. 426 p.

RILEY, Pamela (1988) «Road Culture of International Long-Term Budget Travelers", Annals of Tourism Research, vol. 15, p. 313-328.

ROTH, Eli (2005) Hostel, film, États-Unis : Hostel LLC, DVD, 94 min.

SCHEYVENS, Regina (2002) «Backpacker Tourism and Third World Development», Annals of Tourism Research, vol. 29, p. 144-164.

SORENSEN, Anders (2003) «Backpacker ethnography», Annals of Tourism Research, vol. 30, n 4, p. 847-867.

SPREITZHOFER, Gunter (1998) «Backpacking Tourism in Southeast Asia», Annals of Tourism Research, vol. 25, p. 979-981.

TEAS, Jane (1988), «"I’m Studying Monkeys; What do You Do?”: Youth and Travelers in Nepal», Kroeber Anthropological Society Papers, vol. 67-68, p. 35-41.

TEO, Peggy et Sandra LEONG (2006) «A Postcolonial Analysis of Backpacking", Annals of Tourism Research, vol. 33, nº 1, p. 109-131.

TSAUR, Sheng-Hshiung; Gwo-Hshiung TZENG et Gwo-Ching WANG (1997) «Evaluating tourist risks from fuzzy perspectives», Annals of Tourism Research, vol. 24, $\mathrm{n}^{\circ}$ 4, p. 796-812.

URIELY, Natan; Yuval YONAY et Dalit SIMCHAI (2002) «Backpacking Experiences: A Type and Form Analysis», Annals of Tourism Research, vol. 29, p. 520-538.

VANCE, Paul (2004) «Backpacker transport choice: A conceptual framework applied to New Zealand», DANS RICHARDS, Greg et Julie WILSON (sous la direction de), The global nomad, p. 237-252. Clevedon : Channel View.

VOGT, John (1976) «Wandering: Youth and Travel Behaviour », Annals of Tourism Research, vol. 4, p. 25-41. 